\title{
X-ray edge problem of graphene
}

\author{
S.-R. Eric Yang \\ Department of Physics, Korea University, Seoul, Korea \\ Hyun C. Lef* \\ Department of Physics and Basic Science Research Institute, Sogang University, Seoul, Korea
}

(Dated: November 6, 2018)

\begin{abstract}
The X-ray edge problem of graphene with the Dirac fermion spectrum is studied. At half-filling the linear density of states suppresses the singular response of the Fermi liquid, while away from half-filling the singular features of the Fermi liquid reappear. The crossover behavior as a function of the Fermi energy is examined in detail. The exponent of the power-law absorption rate depends both on the intra- and inter-valley scattering, and it changes as a function of the Fermi energy, which may be tested experimentally.
\end{abstract}

PACS numbers: 72.15.Qm,73.20.-r,73.23-b,78.20.-e,78.70.Dm

Keywords: graphene,orthogonality catastrophe,Dirac Fermions,excitonic process

\section{INTRODUCTION}

Recently the graphenes are being studied intensively mainly due to its novel transport properties such as quantum Hall effect, $1,2,3,4$ Many of such novel properties are attributed to the Dirac fermion band structure of a graphene. ${ }^{5.6}$ The density of states (Dos) is linear in energy, and vanishes at the Fermi energy at half-filling. Therefore, many properties of graphene at half-filling are expected to be markedly different from those of the ordinary Fermi liquid (FL) which has a finite Dos at the Fermi energy. However, away from half-filling, the Dos of graphene does not vanish at the Fermi energy although it can be very small, and some features of FL are expected to emerge.

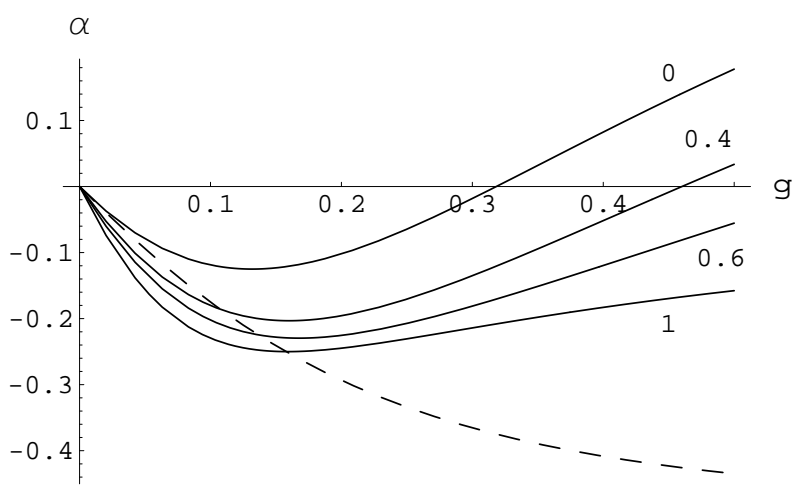

FIG. 1: The dependence of power-law exponent $\alpha$ of graphene on the coupling constant $g=\rho V_{0}$ for $N_{c}=8$ (see text for definitions). From top to bottom, $\frac{V_{1}}{V_{0}}=0,0.4,0.6,1$. Note that the dependence on $g$ implies the dependence on the the Fermi energy. The dashed curve is for the Fermi liquid.

The problem of the transient optical response of FL has a long and interesting history $7,8,9,10,11$ The high-energy $\mathrm{X}$-ray creates a deep core hole, and as a result the Fermi sea of conduction electrons reacts to a time-dependent localized potential. In terms of the optical response the conduction electrons react in two distinctive ways: the excitonic processes 8 and the orthogonality catastrophe $(\mathrm{OC})^{9}$. For an attractive potential stemming from the created hole, the excitonic processes tend to enhance the optical response, while OC effects suppress the optical response for either repulsive or attractive potential. It is crucial to include these two effects on equal footing. For Fermi liquids with a short-range core hole potential $V(\vec{r})=-V \delta(r)$, the absorption intensity which is asymptotically exact near threshold takes the following form. 10

$$
I(\omega) \sim \theta\left(\omega-\omega_{T}^{*}\right) \rho\left(\frac{\omega-\omega_{T}^{*}}{E_{c}}\right)^{\alpha_{\mathrm{FL}}},
$$

where $\omega_{T}^{*}$ is the threshold energy which is the energy gap between the core level and the Fermi energy of FL with corrections from particle-hole interaction. $\theta(\omega)$ is the step function. The power-law exponent is given by ${ }^{12}$

$$
\alpha_{\mathrm{FL}}=-2 \frac{\delta}{\pi}+N_{s}\left(\frac{\delta}{\pi}\right)^{2}, \quad \delta=\tan ^{-1}(\pi \rho V) .
$$

The constants $\rho, E_{c}, N_{s}, \delta$ are the Dos at the Fermi energy, a high-energy cutoff, the spin degeneracy 2 , and the scattering phase shift, respectively.

The X-ray edge problem and the OC have the universal character of the dynamic quantum impurity in gapless host system, and have been studied in diverse physical systems. The tunneling junction with a localized impurity having multiple scattering channel has been studied by Matveev and Larkin 13 using an analogy with the Xray edge problem. The X-ray edge problem and the OC in one-dimensional Luttinger liquid have been studied extensively in Ref. 14, 15, 16, 17, 18. Khveshchenko and Anderson ${ }^{19}$ studied the X-ray edge problem of the tomographic Luttinger liquid which has a vanishing Dos at Fermi energy. Patton and Geller ${ }^{20}$ have obtained the anomalous tunneling Dos of strongly correlated electron systems by mapping the original Hamiltonian to the one to which the method of X-ray edge problem can be applied. We also note that the Kondo physics belongs to 
the same class as the X-ray edge problem. A subclass of the Kondo physics problems where the conduction electrons have power law Dos (soft gap) at Fermi energy has been studied very extensively mainly in the context of the anisotropic superconductors and the dynamical mean field theory: ${ }^{21,22,23}$

At a sufficiently large filling graphene is expected to exhibit FL characters. On the other hand, at and near halffilling the conventional approaches to the optical response of FL cannot be applied directly due to the qualitative differences in band structure between graphene and FL. For example, the bosonization method 11,24 cannot be applied because the local Green function of graphene cannot be represented by that of one-dimensional chiral fermions $\stackrel{24}{2}$ Another new feature of graphene, not present in the ordinary Fermi liquid, is the inter-valley scattering. Here we employ the method of functional integrals to overcome these problems, and integrate out all conduction electron degrees of freedom except for the one at the position of the immobile hole. As far as the local response is concerned, the resulting local action contains all the necessary information. ${ }^{25,26}$ The analysis of the local action based on path integral approach enables us to study the FL and the graphene in a parallel way, highlighting the similarities and the differences at the same time.

In this paper we report theoretical results on the X-ray edge problem of graphene based on the massless Dirac fermion model. We find that the X-ray absorption intensity has the following power-law dependence $\left(\omega>\omega_{T}^{*}\right)$

$$
I(\omega) \sim \rho\left[\left(\frac{\omega-\omega_{T}^{*}}{E_{c}}\right)^{\alpha}+\frac{E_{c}}{\mu}\left(\frac{\omega-\omega_{T}^{*}}{E_{c}}\right)^{1+N_{c}(\delta / \pi)^{2}}\right]
$$

with the exponent

$$
\alpha=-2 \frac{\delta_{+}}{\pi}+N_{c}(\delta / \pi)^{2}, \quad \delta^{2}=\frac{1}{2}\left(\delta_{+}^{2}+\delta_{-}^{2}\right),
$$

where $\delta_{ \pm}=\tan ^{-1}\left[\pi \rho\left(V_{0} \pm V_{1}\right)\right]$ with the short-range intra- and inter-valley scattering strengths $V_{0}$ and $V_{1}$, respectively. In the basis of Dirac fermions (see below), the scattering potential takes the form of the following $4 \mathrm{x} 4$ matrix which is consistent with the symmetry of graphene $\stackrel{27}{2}$

$$
V_{\text {scat }}(\vec{r})=\delta(\vec{r})\left(V_{0} \mathrm{I}_{4}+V_{1} \sigma_{x} \otimes \tau_{x}\right) \equiv \delta(\vec{r}) \tilde{V} .
$$

The combination $V_{0} \pm V_{1}$ in the phase shift $\delta_{ \pm}$is nothing but the eigenvalue of the scattering matrix $\tilde{V}$, and the Green functions are also naturally decomposed with the corresponding eigenvectors, see Eq.(22). The exponent $\alpha$ of the first term in Eq.(3) can be negative, which implies that the absorption intensity is singular at the threshold energy $\omega_{T}^{*}$. On the other hand, the second term is non-singular since the corresponding exponent is always positive. However, near half filling the second term of Eq.(3) dominates since $\rho \propto \mu$, while the first term dominates away from half filling (the Fermi energy $\mu$ is zero at half-filling). The exponent $\alpha$ contains $N_{c}=4$ (8 if spin included) in contrast with the Fermi liquid case of $N_{s}=2$. Since the phase shifts $\delta_{ \pm}$depends on $\rho$, the exponent $\alpha$ also depends on the Fermi energy $\mu$, see Fig 1. In graphene the electron density can be varied up to $10^{13} \mathrm{~cm}^{-2}$ by controlling the gate potentials, $, 1,2,3$ and this opens up the possibility of the systematic measurement of the dependence of the exponent $\alpha$ on the electron density. As we discuss in Sec. VII our result for the X-ray absorption intensity has several other interesting experimental consequences.

This paper is organized as follows: In Sec. II we describe our model Hamiltonian. In Sec. III its local action is formulated within path integral formalism. The Green functions of Dirac fermion and the core hole are derived in Secs. IV and V. Using these Green functions we derive the X-ray absorption intensity in Sec. VI. The discussions are given in Sec. VII.

\section{MODEL HAMILTONIAN FOR GRAPHENE}

Graphene can be described by the following tightbinding Hamiltonian of $\pi$-bands $\stackrel{28}{2}$

$$
\mathcal{H}_{0}=-t \sum_{\mathbf{R}, \mathbf{r}_{i}}\left[c_{A}^{\dagger}(\mathbf{R}) c_{B}\left(\mathbf{R}+\mathbf{r}_{i}\right)+\text { h.c. }\right],
$$

where $A, B$ denote sublattices, and $\mathbf{R}$ (with $\mathbf{r}_{i}$ ) spans a honeycomb lattice. The hopping parameter is $t=$ $-3.033 \mathrm{eV}$, and the basis vectors are given by $\mathbf{a}_{1}=$ $\ell\left(\frac{\sqrt{3}}{2},-\frac{1}{2}\right), \mathbf{a}_{2}=\ell(0,1)$. The lattice spacing is $\ell=2.46 \AA$. Each site of A sublattice is connected to three B sublattice sites: $\mathbf{r}_{1}=\ell\left(\frac{1}{2 \sqrt{3}}, \frac{1}{2}\right), \quad \mathbf{r}_{2}=\ell\left(\frac{1}{2 \sqrt{3}},-\frac{1}{2}\right), \quad \mathbf{r}_{3}=$ $\ell\left(-\frac{1}{\sqrt{3}}, 0\right)$. The energy eigenvalue is given by $E_{ \pm}(\mathbf{k})=$ $\pm t|\Lambda(\mathbf{k})|$, where $\Lambda(\mathbf{k}) \equiv e^{i \mathbf{k} \cdot \mathbf{r}_{1}}+e^{i \mathbf{k} \cdot \mathbf{r}_{2}}+e^{i \mathbf{k} \cdot \mathbf{r}_{3}}$. Two bands touch at 6 points located along the corners of Brillouin zone, and among them only two are distinct: $\mathbf{K}_{+}=\frac{4 \pi}{\sqrt{3} \ell}\left(\frac{1}{2}, \frac{1}{2 \sqrt{3}}\right)$ and $\mathbf{K}_{-}=-\mathbf{K}_{+}$. These two points will be denoted as $K$ and $K^{\prime}$, respectively. From now on we will set the lattice constant $\ell=1$ and $\hbar=1$.

At low energy the above Hamiltonian reduces to the effective Hamiltonian of the massless Dirac fermion, which is valid within a few $\mathrm{eV}$ of the $K$ and $K^{\prime}$ points ${ }^{5}, 6$. In a 4-component notation involving two from sublattice $A, B$ and two from valley indices $\mathbf{K}_{ \pm}$, the Hamiltonian has the form ${ }^{29}$

$$
\mathrm{H}_{0}=\int d^{2} x \Psi^{\dagger}(x)\left(v\left(-i \partial_{x}\right) \alpha^{x}+v\left(-i \partial_{y}\right) \alpha^{y}\right) \Psi(x),
$$

where $v \propto t$ is the velocity of Dirac fermion and $\Psi=$ $\left[c_{A+}, c_{B+}, c_{A-}, c_{B-}\right]^{T}$ is the 4-component spinor (spin indices suppressed). The gamma matrices are $\alpha^{x}=$ $\sigma_{x} \otimes \mathrm{I}_{2}, \quad \alpha^{y}=\sigma_{y} \otimes\left(-\tau_{3}\right), \quad \beta=\sigma_{z} \otimes \mathrm{I}_{2}$, and they satisfy the standard gamma matrix algebra $\left\{\alpha^{i}, \alpha^{j}\right\}=2 \delta^{i j}$. $\tau_{i}, \sigma_{i}$ are two independent copies of Pauli matrices acting on the sublattice and the valley space, respectively. 
The Matsubara Green function of Dirac fermion with Fermi energy $\mu$ is given by

$$
\begin{aligned}
G_{a b}(i \epsilon, \mathbf{k}) & =-\left\langle\Psi_{a}(i \epsilon, \mathbf{k}) \Psi_{b}^{\dagger}(i \epsilon, \mathbf{k})\right\rangle \\
& =\left(\frac{1}{i \epsilon+\mu-v \sum_{j=x, y} k^{j} \alpha^{j}}\right)_{a b} \\
& =\left(\frac{i \epsilon+v \sum_{j} k^{j} \alpha^{j}}{(i \epsilon+\mu)^{2}-v^{2} \mathbf{k}^{2}}\right)_{a b},
\end{aligned}
$$

where $a, b$ are the collective sublattice/valley indices, $i \epsilon$ is the imaginary frequency, and $k^{j}$ is the $\mathrm{j}$-th component of wavevector $\mathbf{k}$. In the last line of the above equation the gamma matrix algebra has been used.

In our model the core potential is taken to be isotropic since the core hole belongs to the $s$-level. Also, it is assumed that the core potential is screened instantly, and this assumption should be valid ${ }^{7}$ near the threshold energy $\omega_{T}^{*}$. Thus the deep core hole can be described by

$$
\mathrm{H}_{\mathrm{int}}=d^{\dagger} d \Psi^{\dagger}(x=0) \tilde{V} \Psi(x=0)
$$

with the scattering potential $\tilde{V}$ given by Eq. (15). $d$ is the annihilation operator of the deep core electron and its Hamiltonian is given by $E_{d} d^{\dagger} d$, where $E_{d}<0$ is the core level energy. The (bare) Matsubara Green function of the core hole is given by

$$
D_{0}(i \epsilon)=\frac{1}{i \epsilon+\mu-E_{d}} .
$$

The interaction with X-ray is described by

$$
\mathrm{H}_{X}=\sum_{a, \mathbf{k}} M_{a, \mathbf{k}} \Psi_{a}^{\dagger}(\mathbf{k}) d e^{-i \omega t}+\text { H.C.. }
$$

$M_{a, \mathbf{k}}$ is the X-ray matrix element. Usually the matrix element is very weakly dependent on $\mathbf{k}$ and its dependence will be ignored. The dependence on the index $a$ will be also ignored. In this paper the interaction between Dirac fermions is not taken into account.

\section{FORMULATION IN TERMS OF LOCAL ACTION}

The local Matsubara Green function of Dirac fermions of graphene with the Fermi energy $\mu$ is given by

$$
\begin{aligned}
& G_{a b}\left(\tau-\tau^{\prime}\right) \equiv-\left\langle\Psi_{a}(\vec{x}=0, \tau) \Psi_{b}^{\dagger}\left(\vec{x}=0, \tau^{\prime}\right)\right\rangle \\
& =\frac{1}{\beta} \sum_{\epsilon} \int \frac{d^{2} \mathbf{k}}{(2 \pi)^{2}} e^{-i \epsilon\left(\tau-\tau^{\prime}\right)} G_{a b}(i \epsilon, \mathbf{k}) \\
& =\delta_{a b} \frac{1}{\beta} \sum_{\epsilon} \int \frac{d^{2} \mathbf{k}}{(2 \pi)^{2}} \frac{e^{-i \epsilon\left(\tau-\tau^{\prime}\right)}(i \epsilon+\mu)}{(i \epsilon+\mu)^{2}-v^{2} \mathbf{k}^{2}} .
\end{aligned}
$$

Note that in the last line of the above equation, the term depending on momentum linearly $\left[\alpha^{j} k^{j}\right.$, see Eq.(8) ] vanished upon wavevector integration. If $\left|\tau-\tau^{\prime}\right| \gg \tau_{c}$, where $\tau_{c}$ is a short time cutoff, Eq.(12) becomes at zero temperature

$$
\begin{aligned}
G_{a b}\left(\tau-\tau^{\prime}\right) & =\delta_{a b} G=\delta_{a b}\left(G_{\mathrm{FL}}+G_{\mathrm{D}}\right), \\
G_{\mathrm{D}}\left(\tau-\tau^{\prime}\right) & =-\frac{1}{4 \pi v^{2}} \frac{1}{\left(\tau-\tau^{\prime}\right)^{2}}+\frac{\theta\left(\tau^{\prime}-\tau\right)}{4 \pi v^{2}} \frac{e^{-\mu\left(\tau^{\prime}-\tau\right)}}{\left(\tau-\tau^{\prime}\right)^{2}} \\
G_{\mathrm{FL}}\left(\tau-\tau^{\prime}\right) & =-\frac{\rho}{\tau-\tau^{\prime}},
\end{aligned}
$$

where

$$
\rho \equiv \frac{\mu}{4 \pi v^{2}}
$$

which plays the role of Dos at the Fermi energy. Note that it is proportional to $\mu$. Thus the local Green function consists of the Fermi liquid component $G_{\mathrm{FL}}$ and the pure Dirac component $G_{\mathrm{D}}$. In the long time/low energy limit the Dirac component is much less singular than the FL component. In fact, the direct Feynman diagram calculations at half-filling show the logarithmic singularities originating from $G_{\mathrm{FL}}$ do not exist, so that the perturbation theory converges well at low energy. For details, see Sec. VII.

To obtain the action of the local degrees of freedom we have to integrate out the bulk degrees of freedom except for the one at $\vec{x}=0$. For this step, we use the method of Dirac delta function in Grassman variable in the following form

$$
\begin{aligned}
1 & =\int D\left[\eta_{a}, \bar{\eta}_{a}\right] \prod_{a=1}^{4} \delta\left(\eta_{a}-\Psi_{a, x=0}\right) \delta\left(\bar{\eta}_{a}-\Psi_{a, x=0}^{\dagger}\right) \\
& =\int D\left[\eta_{a}, \bar{\eta}_{a}, \lambda_{a}, \bar{\lambda}_{a}\right] \exp \left[\sum_{a} \bar{\lambda}_{a}(\tau)\left(\eta_{a}(\tau)-\Psi_{a}(0, \tau)\right)\right. \\
& \left.+\left(\bar{\eta}_{a}(\tau)-\Psi_{a}^{\dagger}(0, \tau)\right) \lambda_{a}(\tau)\right]
\end{aligned}
$$

where $\lambda_{a}$ is a Grassman Lagrangian multiplier. The local action of the local degrees of freedom $\eta_{a}(\tau), \bar{\eta}_{a}(\tau)$ can be obtained by integrating out $\Psi_{a}$ and $\lambda_{a}$ successively.

The resulting local action of $\eta_{a}$ and $d$ in imaginary time is

$$
\begin{aligned}
& S[\eta, d]=-\sum_{a, b} \int d \tau d \tau^{\prime} \bar{\eta}_{a}(\tau) G_{a b}^{-1}\left(\tau-\tau^{\prime}\right) \eta_{b}\left(\tau^{\prime}\right) \\
& +\int d \tau \bar{d}(\tau) d(\tau) \bar{\eta}(\tau) \tilde{V} \eta(\tau)+\int d \tau \bar{d}\left(\partial_{\tau}-\omega_{T}\right) d
\end{aligned}
$$

where $\omega_{T}=\mu-E_{d}$ is the (bare) threshold energy and $G_{a b}^{-1}$ is the inverse of the local Green function Eq.(12) of the Dirac fermion. The local action Eq.(16) is of a very general form, so that it can be applied to other cases with different band structures, including FL. The specific band structure is reflected only in the local Green function $G_{a b}\left(\tau-\tau^{\prime}\right)$. 


\section{THE GREEN FUNCTION OF DIRAC FERMION}

In the presence of a following time-dependent potential

$$
\widehat{V}(\tau)=-\tilde{V} \theta\left(\tau_{1}-\tau\right) \theta\left(\tau-\tau_{2}\right)
$$

the local Matsubara Green function of Dirac fermions is given by $\left(\xi, \xi^{\prime}\right.$ are imaginary times)

$$
g\left(\xi-\xi^{\prime}\right)=\left\langle\xi\left|\frac{1}{G^{-1}-\widehat{V}}\right| \xi^{\prime}\right\rangle
$$

which is a $4 \times 4$ matrix Green function, and this Green function encapsulates the excitonic contribution to the $\mathrm{X}$-ray response function. From the above definition of $g$ one can show that it satisfies the following (matrix) integral equation.

$$
g\left(\xi, \xi^{\prime}\right)=G\left(\xi-\xi^{\prime}\right)+\int_{\tau_{2}}^{\tau_{1}} d \tau G(\xi-\tau)(-\tilde{V}) g\left(\tau, \xi^{\prime}\right)
$$

where $G$ is the bare local Green function, Eq. 13), which is diagonal. To solve Eq.(19) we choose an ansatz

$$
g=g_{0} \mathrm{I}_{4}+g_{1} \sigma_{x} \otimes \tau_{x}
$$

and take the combinations of $g_{0} \pm g_{1}$. Then it can be shown that each of $g_{0} \pm g_{1}$ satisfies the following equation

$$
\left(g_{0} \pm g_{1}\right)=G+\left(-V_{0} \mp V_{1}\right) \int_{\tau_{2}}^{\tau_{1}} d \tau G(\xi-\tau)\left(g_{0} \pm g_{1}\right) \text {. }
$$

When $G$ is of the FL form $G_{\mathrm{FL}}(\tau)=-\rho / \tau$, the solution of Eq. (21) which is asymptotically exact in the long time limit was obtained by Nozières and De Dominicis (ND) ${ }^{10}$ :

$$
\begin{aligned}
\left(g_{0} \pm g_{1}\right)^{\mathrm{ND}}\left(\xi, \xi^{\prime}\right) & =\left[\cos ^{2} \delta_{ \pm}\right] G_{\mathrm{FL}}\left(\xi-\xi^{\prime}\right) \\
& \times\left[\frac{\left(\xi-\tau_{2}\right)\left(\tau_{1}-\xi^{\prime}\right)}{\left(\tau_{1}-\xi\right)\left(\xi^{\prime}-\tau_{2}\right)}\right]^{\frac{\delta_{ \pm}}{\pi}},
\end{aligned}
$$

with the scattering phase shift given by

$$
\delta_{ \pm}=\tan ^{-1}\left[\pi\left(V_{0} \pm V_{1}\right) \rho\right] .
$$

This result cannot be obtained perturbatively. In view of the fact $G=G_{\mathrm{FL}}+G_{\mathrm{D}}$, the Green functions $g_{0,1}$ can be also written as a sum of

$$
g_{0,1}=g_{0,1}^{\mathrm{ND}}+\delta g_{0,1}
$$

where $\delta g_{0,1}$ can be computed perturbatively since $G_{\mathrm{D}}$ is much less singular than $G_{\mathrm{FL}}$ in the long time limit. Using the above form of $g_{0,1}$ in Eq.(21) and carrying out the integrals carefully we find that $\delta g_{0,1} \approx A_{0,1} g_{0,1}^{\mathrm{ND}}+B_{0,1} G_{\mathrm{D}}$ where $A_{0,1}$ are small multiplicative renormalization constants of the order of $V_{0,1} / t$ and $B_{0,1}$ are also multiplicative renormalization constants. We define, for convenience, new Green functions $g_{0,1}^{\mathrm{FL}}=g_{0,1}^{\mathrm{ND}}+A_{0,1} g_{0,1}^{\mathrm{ND}}$, and $g_{0,1}^{\mathrm{D}}=B_{0,1} G_{\mathrm{D}}$. Note $g_{0,1}^{\mathrm{FL}}$ vanish as $\mu \rightarrow 0$ since they are proportional to $G_{\mathrm{FL}}$, while $g_{0,1}^{\mathrm{D}}$ do not. The explicit forms of these new Green functions are

$$
\begin{aligned}
g_{0,1}^{\mathrm{FL}}\left(\tau_{1}-\tau_{2}\right) & \sim \frac{1}{2} \frac{(-\rho) \cos ^{2} \delta_{+}}{\tau_{1}-\tau_{2}}\left(\frac{\tau_{1}-\tau_{2}}{\tau_{c}}\right)^{\frac{2 \delta_{+}}{\pi}} \\
& \pm \frac{1}{2} \frac{(-\rho) \cos ^{2} \delta_{-}}{\tau_{1}-\tau_{2}}\left(\frac{\tau_{1}-\tau_{2}}{\tau_{c}}\right)^{\frac{2 \delta_{-}}{\pi}}, \\
g_{0}^{\mathrm{D}}\left(\tau_{1}-\tau_{2}\right) & \sim-\frac{1}{4 \pi v^{2}} \frac{1}{\left(\tau_{1}-\tau_{2}\right)^{2}}\left(1+\pi \frac{V_{0}}{4 \pi v^{2}} \frac{1}{\tau_{c}}\right), \\
g_{1}^{\mathrm{D}}\left(\tau_{1}-\tau_{2}\right) & \sim-\frac{1}{4 \pi v^{2}}\left[\pi \frac{V_{1}}{4 \pi v^{2}} \frac{1}{\tau_{c}}\right] \frac{1}{\left(\tau_{1}-\tau_{2}\right)^{2}},
\end{aligned}
$$

where $\rho$ is to be understood as the renormalized Dos at the Fermi energy. From these it follows that

$$
g_{0,1} \approx g_{0,1}^{\mathrm{FL}}+g_{0,1}^{\mathrm{D}}
$$

\section{CORE-HOLE GREEN FUNCTIONS}

The photoemission spectra is related to the core hole Green function which captures the physics of OC.

$$
D\left(\tau_{1}, \tau_{2}\right)=\left\langle T_{\tau} \bar{d}\left(\tau_{1}\right) d\left(\tau_{2}\right)\right\rangle .
$$

At temperature much lower than $\omega_{T}$ this Green function is non-vanishing only for $\tau_{1}>\tau_{2}$. Carrying out the path integral over $d$ in Eq.(16) we obtain

$$
D\left(\tau_{1}, \tau_{2}\right)=\frac{\int D[\eta] e^{-S_{\eta}}\left\langle\tau_{2}\left|\frac{1}{\partial_{\tau}+\bar{\eta} \tilde{V} \eta}\right| \tau_{1}\right\rangle}{\int D[\eta] e^{-S_{\eta}}}
$$

where $S_{\eta}$ is the first term of Eq.(16). In fact, a term $\frac{\tilde{V}}{2} \delta\left(\tau-\tau^{\prime}\right)$ should have been included in $S_{\eta}$. It stems from the determinant of $d$-path integral ${ }^{30}$, but it turns out not to affect the long-time behavior, so has been dropped. $\left\langle\tau_{2}\left|\frac{1}{\partial_{\tau}+\bar{\eta} \tilde{V} \eta}\right| \tau_{1}\right\rangle$ is Green function (1st order in time) in the presence of external potential $\bar{\eta} \tilde{V} \eta$, which is easily calculated to be $\exp \left[-\int_{\tau_{2}}^{\tau_{1}} d \tau^{\prime} \bar{\eta}(-\tilde{V}) \eta\right]$. Now the path integral over $\eta$ can be done. Recalling $G=G_{\mathrm{FL}}+G_{\mathrm{D}}$, the result of the path integral can be expressed as

$$
D\left(\tau_{1}, \tau_{2}\right)=e^{\operatorname{Tr} \ln \left(1-G_{\mathrm{FL}} \widehat{V}\right)} e^{\operatorname{Tr} \ln \left(1-\frac{G_{\mathrm{D}} \hat{V}}{1-G_{\mathrm{FL}} \hat{V}}\right)} .
$$

The first factor of Eq.(29) is the core hole Green function of the Fermi liquid component, and this can be calculated exactly by using the ND solution Eq.(22) 10,24 The second factor does not generate any infrared singularities and it can be evaluated by a perturbative expansion in $\widehat{V}$. Expanding the second factor up to the second order in $\widehat{V}$ we obtain

$$
\begin{aligned}
D\left(\tau_{1}, \tau_{2}\right) & \sim e^{-\omega_{T}^{*}\left(\tau_{1}-\tau_{2}\right)} \frac{1}{\left(\frac{\tau_{1}-\tau_{2}}{\tau_{c}}\right)^{N_{c}}(\delta / \pi)^{2}} \\
& \times \exp \left[-\frac{N_{c}}{4}\left(\frac{V_{0}^{2}+V_{1}^{2}}{\left(4 \pi v^{2}\right)^{2}}\right) \frac{1}{\left(\tau_{1}-\tau_{2}\right)^{2}}\right],
\end{aligned}
$$


where $\omega_{T}^{*}$ is the renormalized threshold energy ${ }^{7}$ and $\delta$ is the exponent given in Eq.(4). $N_{c}=4$ is the number of the species of Dirac fermions, and its presence originates from the trace over spinor space. The result Eq.(30) depends critically on band filling. At half-filling we have $\delta=0$, and the correction from the scattering is negligible in the long time limit. But away from the half-filling the power law dependence dominates. Eq.(30) also implies that the overlap between ground state wavefunctions with and without the core hole is given by

$$
|\langle 0 \mid V\rangle| \sim e^{+\frac{1}{2} N_{c}\left(\frac{\delta}{\pi}\right)^{2} \ln \left(\omega_{\min } \tau_{c}\right)-\text { const. }\left(\omega_{\min } \tau_{c}\right)^{2}},
$$

where $\omega_{\min }$ is the $\pi v / L$ with the system size $L$. At halffilling the first term in the exponent, which is of the FL type, vanishes since $\delta=0$. However, the second term is finite and the overlap becomes almost independent of the system size. This is is consistent with the result of Ref.[31] which was obtained numerically.

The hole spectral function can be obtained from $(\zeta$ is an infinitesimally small positive number)

$$
A_{h}(\omega)=-\operatorname{Im}\left[\left.\int d \tau e^{i \omega \tau} D(\tau)\right|_{i \omega \rightarrow \omega+i \zeta}\right] .
$$

In the long time limit (or in the vicinity of $\omega=\omega_{T}^{*}$ ),

$$
\begin{aligned}
& A_{h}(\omega) \sim \theta\left(\omega-\omega_{T}^{*}\right) \sin \left[\pi N_{c}(\delta / \pi)^{2}\right]\left(\frac{\tau_{c} c_{1}}{\left[\left(\omega-\omega_{T}^{*}\right) \tau_{c}\right]^{1-N_{c}\left(\frac{\delta}{\pi}\right)^{2}}}\right. \\
& \left.-c_{2} \tau_{c}^{-1}\left[\left(\omega-\omega_{T}^{*}\right) \tau_{c}\right]^{1+N_{c}\left(\frac{\delta}{\pi}\right)^{2}}\right),
\end{aligned}
$$

where $(\Gamma(x)$ is the Gamma function)

$$
\begin{aligned}
& c_{1}=\Gamma\left(1-N_{c}(\delta / \pi)^{2}\right), \\
& c_{2}=\left(\frac{V_{0}^{2}+V_{1}^{2}}{\left(4 \pi v^{2}\right)^{2}}\right) \frac{\frac{N_{c}}{4} \Gamma\left(1-N_{c}(\delta / \pi)^{2}\right)}{\left(1+N_{c}(\delta / \pi)^{2}\right) N_{c}(\delta / \pi)^{2}} .
\end{aligned}
$$

The above expressions of $c_{1,2}$ are valid as long as $1>$ $N_{c}(\delta / \pi)^{2}$. If $N_{c}(\delta / \pi)^{2} \geq 1$, the $\tau$-integral of Eq.(32) diverges in the short time limit $\tau \rightarrow 0$. However, we have to remember that the expression Eq.(30) of $D(\tau)$ is valid asymptotically only in the long time limit. Therefore, this divergence is not physically relevant, and it can be treated by introducing an explicit short time cutoff in the integral or by an analytic continuation of Gamma functions appearing in $c_{1}$ and $c_{2}$.

At half-filling we have $\delta=0$, and the core-hole Green function Eq.(30) becomes

$D\left(\tau_{1}, \tau_{2}\right) \sim e^{-\omega_{T}^{*}\left(\tau_{1}-\tau_{2}\right)} \exp \left[-\frac{N_{c}}{4}\left(\frac{V_{0}^{2}+V_{1}^{2}}{\left(4 \pi v^{2}\right)^{2}}\right) \frac{1}{\left(\tau_{1}-\tau_{2}\right)^{2}}\right]$.

Expanding the exponent of Eq.(35) in the long time limit we obtain

$$
\begin{aligned}
A_{h, \delta=0}(\omega) & \sim \pi \delta\left(\omega-\omega_{T}^{*}\right) \\
& -\theta\left(\omega-\omega_{T}^{*}\right)\left(\omega-\omega_{T}^{*}\right)\left(\frac{V_{0}^{2}+V_{1}^{2}}{\left(4 \pi v^{2}\right)^{2}}\right) .
\end{aligned}
$$

Comparing with the result of Eq.(33) $(\delta \neq 0)$, we find that the anomalous power-law dependence has disappeared.

\section{X-RAY RESPONSE FUNCTION}

This is given by

$$
F\left(\tau_{1}, \tau_{2}\right)=\sum_{a, b}\left\langle T_{\tau} \bar{d}\left(\tau_{1}\right) \eta_{a}\left(\tau_{1}\right) \bar{\eta}_{b}\left(\tau_{2}\right) d\left(\tau_{2}\right)\right\rangle,
$$

which is also non-vanishing only for $\tau_{1}>\tau_{2}$. To compute this response function it is convenient to introduce the external source $J_{a}$ for $\eta_{a}, S_{J}=-\int d \tau\left(\sum_{a} \bar{J}_{a} \eta_{a}+\bar{\eta}_{a} J_{a}\right)$, into the local action. Carrying out $d$-integration and $J$ differentiation we arrive at

$$
\begin{aligned}
F\left(\tau_{1}, \tau_{2}\right) & =\left.\sum_{a, b} \frac{\frac{\delta}{\delta J_{b}\left(\tau_{2}\right)} \frac{\delta}{\delta J_{a}\left(\tau_{1}\right)} \int D[\eta] e^{-S[\eta]-S_{J}-\int d \tau \bar{\eta} \widehat{V} \eta}}{\int D[\eta] e^{-S[\eta]}}\right|_{J \rightarrow 0} \\
& =(-1) \frac{\operatorname{Det}\left[G^{-1}-\widehat{V}\right]}{\operatorname{Det}\left[G^{-1}\right]} \sum_{a, b} g_{a b}\left(\tau_{1}-\tau_{2}\right),
\end{aligned}
$$

where $g_{a b}\left(\tau_{1}-\tau_{2}\right)$ is the (matrix) local Green function of the Dirac fermion of Eq. (18). We also note that $\frac{\operatorname{Det}\left[G^{-1}-\widehat{V}\right]}{\operatorname{Det}\left[G^{-1}\right]}$ is the core hole Green function of Eq. (29). The path integral formulation automatically yields that the excitonic effect (represented by $g_{a b}$ ) and the OC (represented by the core hole Green function) contribute in multiplicative way. Therefore, we obtain

$$
F\left(\tau_{1}-\tau_{2}\right)=-\theta\left(\tau_{1}-\tau_{2}\right) D\left(\tau_{1}-\tau_{2}\right) \sum_{a, b} g_{a b}\left(\tau_{1}-\tau_{2}\right) .
$$

From Eq. (20) it follows that

$$
\begin{aligned}
\sum_{a, b} g_{a b}\left(\tau_{1}-\tau_{2}\right) & =4\left(g_{0}+g_{1}\right) \\
& =4\left[\left(g_{0}^{\mathrm{FL}}+g_{1}^{\mathrm{FL}}\right)+\left(g_{0}^{\mathrm{D}}+g_{1}^{\mathrm{D}}\right)\right] .
\end{aligned}
$$

Now the absorption intensity at $T=0$ can be computed from

$$
I(\omega)=-\frac{1}{\pi} \operatorname{Im}\left[\left.\int_{0}^{\infty} d \tau e^{i \omega \tau} F(\tau)\right|_{i \omega \rightarrow \omega+i \zeta}\right] .
$$

Using Eqs. (25/30/39) we find for the absorption rate

$$
I=I_{\mathrm{FL}}+I_{\mathrm{D}}
$$

where

$$
\begin{aligned}
& I_{\mathrm{FL}}(\omega) \sim d_{\mathrm{FL}} \theta\left(\omega-\omega_{T}^{*}\right) \rho\left(\frac{\omega-\omega_{T}^{*}}{E_{c}}\right)^{-2 \frac{\delta_{+}}{\pi}+N_{c}(\delta / \pi)^{2}} \\
& I_{\mathrm{D}}(\omega) \sim d_{\mathrm{D}} \theta\left(\omega-\omega_{T}^{*}\right) \frac{\rho E_{c}}{\mu}\left(\frac{\omega-\omega_{T}^{*}}{E_{c}}\right)^{1+N_{c}(\delta / \pi)^{2}},
\end{aligned}
$$


with the coefficients

$$
\begin{aligned}
d_{\mathrm{FL}} & =\cos ^{2} \delta_{+} \Gamma\left(2 \frac{\delta_{+}}{\pi}-N_{c}(\delta / \pi)^{2}\right) \sin \left[\pi\left(2 \frac{\delta_{+}}{\pi}-N_{c}(\delta / \pi)^{2}\right)\right], \\
d_{\mathrm{D}} & =\sin \left[N_{c}(\delta / \pi)^{2}\right] \frac{\Gamma\left(1-N_{c}(\delta / \pi)^{2}\right)}{\left[1+N_{c}(\delta / \pi)^{2}\right] N_{c}(\delta / \pi)^{2}} .
\end{aligned}
$$

The above expressions of $d_{\mathrm{FL}}$ and $d_{\mathrm{D}}$ are valid as long as $1>N_{c}(\delta / \pi)^{2}$. In the case of $1 \leq N_{c}(\delta / \pi)^{2}, d_{\mathrm{FL}, \mathrm{D}}$ are given by an analytic continuation of Gamma functions as in the case of the core-hole spectral function. In particular, $d_{\mathrm{FL}}$ is positive even if $2 \frac{\delta_{+}}{\pi}-N_{c}\left(\frac{\delta}{\pi}\right)^{2}$ becomes negative, owing to the identity $1=\Gamma(1-x) \Gamma(x) \sin (\pi x) / \pi$. Note that only the exponent $\delta_{+}$appears in Eq. (42) because the second term of $g_{0,1}^{\mathrm{FL}}\left(\tau_{1}-\tau_{2}\right)$ which has a $\delta_{-}$exponent [see Eq. (25, 40)] is canceled in the summation over channels $a, b$ of Eq.(39). In the above we have assumed that the X-ray matrix element is very weakly dependent on the sublattice and valley structure. Let us compare our result of the exponent of Eq. (42) with that of Ref. 13]. Eq.(9) of Ref. [13] is the leading term of the exact result Eq.(72) of Ref.[10]. In our case only $s$-wave orbital scattering is relevant, and the effect of channels other than the orbital such as valley and sublattice is reflected in the factor $N_{c}$. Therefore, our result is fully consistent with that of Ref.[13].

The $I_{\mathrm{FL}}(\omega)$ vanishes as half-filling is approached since $\rho$ and $\delta_{ \pm} \rightarrow 0$ at the same time, while the $I_{\mathrm{D}}$ part remains finite. The exponent $-2 \frac{\delta_{+}}{\pi}+N_{c}\left(\frac{\delta}{\pi}\right)^{2}$ is a sum of the exponents for the excitonic and OC processes, $-2 \frac{\delta_{+}}{\pi}$ and $N_{c}\left(\frac{\delta}{\pi}\right)^{2}$, respectively. Away from half-filling, if this exponent is negative, $I_{\mathrm{FL}}$ is the dominant term since it diverges as $\omega \rightarrow \omega_{T}$, manifesting the X-ray singularity. The $I_{\mathrm{D}}$ is finite and does not exhibit a singular behavior.

At half-filling, even in the absence of the local potential, the intensity is suppressed by a factor of $\omega-\omega_{T}^{*}$ near threshold, and this is a consequence of the effect of the linear Dos. The linear Dos completely suppresses the excitonic processes near the threshold, and the perturbative treatment is sufficient, as can be checked explicitly by a direct calculation of Feynman diagrams (see below).

\section{DISCUSSIONS}

The singular nature of X-ray edge problem of FL can be traced back to the logarithmic divergence of 1-loop particle-hole polarization function $\underline{7}^{\frac{7}{}}$ Due to these $\log$ arithmic divergences the infinitely many Feynman diagrams should be summed even in the weak coupling region, and the summation leads to the anomalous power law dependence of response functions. Thus to understand the non-singular nature of response functions of graphene at half filling $(\mu=0, \rho=0)$, it suffices to calculate the 1-loop particle-hole polarization function and to show that it is non-singular. The 1-loop polarization function of graphene (at half-filling and at zero temperature) is given by

$$
\begin{aligned}
& \Pi^{(0)}(i \omega)=-\int \frac{d \epsilon}{2 \pi} \int \frac{d^{2} \mathbf{k}}{(2 \pi)^{2}} \operatorname{Tr} \widehat{G}(i \epsilon, \mathbf{k}) D_{0}(i \epsilon-i \omega) \\
& =-N_{c} \int \frac{d \epsilon}{2 \pi} \int \frac{d^{2} \mathbf{k}}{(2 \pi)^{2}} \frac{i \epsilon}{(i \epsilon)^{2}-v^{2} \mathbf{k}^{2}} \frac{1}{i \epsilon-i \omega+\omega_{T}},
\end{aligned}
$$

where $\widehat{G}(i \epsilon, \mathbf{k})$ is the matrix Green function of Eq. (8) at $\mu=0$, and $D_{0}(i \epsilon-i \omega)$ is the bare core hole Green function, given in Eq.(10). In the last line of the above equation the term linear in $\mathbf{k}$ vanishes upon $\mathbf{k}$ integration. The integral is straightforward, and we obtain

$$
\Pi^{(0)}(i \omega) \sim \frac{1}{2 \pi v^{2}}\left[\left(i \omega-\omega_{T}\right) \ln \left(\frac{\omega_{T}-i \omega}{E_{c}}\right)-E_{c}\right],
$$

where $E_{c}$ is an energy cutoff of the order $t$. As demonstrated in the above (after analytic continuation $i \omega \rightarrow$ $\omega+i \zeta)$, the logarithmic singularity near the threshold $\ln \left(\omega_{T}-\omega\right)$ is completely suppressed by the prefactor $\omega-\omega_{T}$ which stems from the linearly vanishing density of states of graphene. In the case of FL, the prefactor $\omega-\omega_{T}$ is replaced by the (finite) density of states $\rho$. Therefore, for graphene at half-filling, the higher order corrections are not necessary if we are only interested in the vicinity of threshold.

It would be interesting to observe in X-ray experiments of a single layer graphene ${ }^{32,33}$ how the exponent $\alpha$ of Eq.(3) depends on the electron density. In graphene the carrier density can be varied up to $10^{13} \mathrm{~cm}^{-2}$ by changing external gates $1.2,3$. This corresponds to a change in the Fermi energy up to a few eV. The typical X-ray energy range is given by the energy difference between the core electron in the $1 s$ level and Dirac fermions residing in the $\pi$ band, which is in the region of $280 \sim 290 \mathrm{eV} \stackrel{28,32}{=}$ One of the key experimental features would be that the absorption spectrum diverges at the threshold energy $\omega_{T}^{*}$, which would correspond to a negative exponent $\alpha$ in the first term of Eq.(3) (the second term of Eq.(3) is zero at the at the threshold energy $\omega=\omega_{T}^{*}$ ). In the Fermi liquid the exponent decreases monotonically with the electron density, while in graphene it decreases initially and then increases and becomes positive, see Fig.1. When the exponent becomes positive the absorption spectrum does not diverge at the threshold. Experimental verification of this change of sign of the exponent in graphene would be intersting. The experimental measurement of $\alpha$ would also provide useful information about the strength of intra and inter valley scattering strengths $V_{0}$ and $V_{1}$.

In this paper, the hole was assumed to be immobile (or of infinite mass) $\underbrace{20,34,35,36}$ The finite mass effect and the finite temperature ${ }^{37}$ are expected to smear out the singular behavior to some extent, and these factors are to be taken into account for the detailed comparison with experimental data. 


\section{Acknowledgments}

This work was supported by the Grant No. R01-2005000-10352-0 from the Basic Research Program of the Ko- rean Science and Engineering Foundation, by the Korea Research Foundation Grant funded by the Korean Government (MOEHRD)(KRF-2005-070-C0044), and by The Second Brain Korea 21 Project.
* the author to whom the correspondences should be addressed: hyunlee@sogang.ac.kr

1 K. S. Novoselov, A. K. Geim, S. V. Morozov, D. Jiang, Y. Zhang, S. V. Dubonos, I. V. Gregorieva, and A. A. Firsov, Science 306, 666 (2004).

2 K. S. Novoselov, A. K. Geim, S. V. Morozov, D. Jiang, M. I. Katnelson, I. V. Gregorieva, S. V. Dubonos, and A. A. Firsov, Nature 438, 197 (2005).

3 Y. Zhang, Y.-W. Tan, H. L. Störmer, and P. Kim, Nature 438, 201 (2005).

4 A. K. Geim and K. S. Novoselov, Nature Materials 6, 183 (2007).

5 J. C. Slonczewski and P.R. Weiss, Phys. Rev. 109, 272 (1958). D.P. DiVincenzo and E.J. Mele, Phys. Rev. B 29, 1685, (1984), C.L. Kane and E.J. Mele, Phys. Rev. Lett,78, 1932 (1997).

6 T. Ando, T. Nakanishi, and R. Saito, J. Phys.Soc.Jpn. 67, 2857 (1998).

7 G. Mahan, Many-Particle Physics, Plenum Publishers, New York (2000).

8 G. Mahan, Phys. Rev. 163, 612 (1967).

9 P. W. Anderson, Phys. Rev. Lett. 18, 1049 (1967)

10 P. Nozières and C. de Dominicis, Phys. Rev. 178, 1097 (1969).

11 K. D. Schotte and U. Schotte, Phys. Rev. 182, 479 (1969).

12 In Ref. 10] the short-time correction to $\delta$ [Eq.(44)] were also included in the form of $\tan \delta=\pi \rho V /(1-\pi \rho V \tan \theta)$. In our paper we will neglect such short-time correction as $\pi \rho V \tan \theta$.

13 K. A. Matveev and A. I. Larkin, Phys. Rev. B 46, 15337 (1992).

14 A. Gogolin, Phys. Rev. Lett. 71, 2995 (1993)

15 T. Ogawa, A. Furusaki, and N. Nagaosa, Phys. Rev. Lett. 68, 3638 (1992)

16 D. K. K. Lee and Y. Chen, Phys. Rev. Lett. 69, 1399 (1992).

17 N. V. Prokofev, Phys. Rev. B 49, 2148 (1994).

18 C. L. Kane, K. A. Matveev, and L. I. Glazman, Phys. Rev. B 49, 2253 (1994).

19 D. V. Khveshchenko and P. W. Anderson, Phys. Rev. B 61, 1658 (2000).
${ }^{20}$ K. R. Patton and M. R. Geller, Phys. Rev. B 72, 125108 (2005); K. R. Patton and M. R. Geller, Phys. Rev. B 73, 125416 (2006).

21 D. Withoff and E. Fradkin, Phys. Rev. Lett. 64, 1835 (1990).

22 R. Bulla and M. Vojta, in Concepts in Electron Correlations, edited by A. C. Hewson and V. Zlatić (Kluwer Academic Publishers, Dordrecht), p. 209.

23 C. Gonzalez-Buxton and K. Ingersent, Phys. Rev. B 57, 14254 (1998)

24 A. Gogolin, A. Nersesyan, and A. Tsvelik, Bosonization and Strongly Correlated Systems, Cambridge University Press, Cambridge (1998).

${ }^{25}$ C. L. Kane and M. P. A. Fisher, Phys. Rev. B 46, 15233 (1992)

26 A. Furusaki and N. Nagaosa, Phys. Rev. B 47, 4631 (1993).

27 M. Igami, T. Nakanishi, T. Ando, J. Phys.Soc.Jpn. 68, 716 (1999).

28 R. Saito, G. Dresselhaus, and M. S. Dresselhaus, Physical Properties of Carbon Nanotubes, Imperial College Press, London (2003).

29 H. Suzuura and T. Ando, Phys. Rev. Lett. 89, 266603 (2002).

30 H. Kleinert, Path Integrals 3rd Edition, World Scientific, Singapore (2005), p. 612.

31 M. Hentschel and F. Guinea, arXiv:0705.0522 (2007).

32 E. Rollings, G.-H. Gweon, S.Y. Zhou, B.S. Mun, J.L. McChesney, B.S. Hussain, A.V. Fedorov, P.N. First, W.A. de Heer, and A. Lanzara, J. Phys. Chem. Sol., 67, 2172, (2006).

33 A. Bostwick, T. Ohta, T. Seyller, K. Horn, and E. Rotenberg, Nature Physics, 3, 36 (2007).

34 T. Uenoyama and L. J. Sham, Phys. Rev. Lett. 65, 1048 (1990).

35 J. Gavoret, P. Nozières, B. Roulet, and M. Combescot, J. Phys. (Paris) 30, 987 (1969).

36 A. E. Ruckenstein and S. Schmitt-Rink, Phys. Rev. B 35, 7551 (1987).

37 K. Ohtaka and Y. Tanabe, Phys. Rev. B 39, 3054 (1989). 